\title{
Research Article \\ Size, Shape, and Material Effects in Ferroelectric Octahedral Nanoparticles
}

\author{
Daopei Zhu $\mathbb{D}^{1}{ }^{1}$ Haocheng Yan $\mathbb{D}^{1},{ }^{1}$ Siyuan Tian $\mathbb{D}^{1},{ }^{1}$ and Zhangli Wang $\mathbb{D}^{2}$ \\ ${ }^{1}$ School of Civil and Surveying \& Mapping Engineering (Nanchang), Jiangxi University of Science and Technology, Nanchang, \\ Jiangxi 330013, China \\ ${ }^{2}$ Gansu Construction Investment (Holdings) Group Corporation Limited, Lanzhou, Gansu 730030, China
}

Correspondence should be addressed to Zhangli Wang; zhangli.wang@hotmail.com

Received 17 April 2021; Accepted 30 June 2021; Published 17 July 2021

Academic Editor: Abdelwahab Omri

Copyright (C) 2021 Daopei Zhu et al. This is an open access article distributed under the Creative Commons Attribution License, which permits unrestricted use, distribution, and reproduction in any medium, provided the original work is properly cited.

Composite materials composed of multiferroelectric nanoparticles in dielectric matrixes have attracted enormous attention for their potential applications in developing future functional devices. However, the functionalities of ferroelectric nanoparticles depend on shapes, sizes, and materials. In this paper, a time-dependent Landau-Ginzburg method has been used and combined with a method as the coupled-physics finite-element-method-based simulations are used to illustrate the polarization behavior in isolated $\mathrm{BaTiO} 3$ or $\mathrm{PbTiO} 3$ octahedral nanoparticles embedded in a dielectric medium, like SrTiO3 (ST, high dielectric permittivity) and amorphous silica (a-SiO2, low dielectric permittivity). The equilibrium polarization topology of the octahedral nanoparticle is strongly affected by the choice of inclusion and the size of matrix materials. Also, there are three equilibrium polarization patterns, i.e., monodomain, vortex-like, and multidomain, because of the various sizes and material parameters combination. There is a critical particle size below which ferroelectricity vanishes in our calculations. This size of the PbTiO3 octahedral nanoparticle is 2.5 and $3.6 \mathrm{~nm}$ for high- and lowpermittivity matrix materials, respectively. However, this size of the BaTiO3 octahedral nanoparticle is $3.6 \mathrm{~nm}$ regardless of the matrix materials.

\section{Introduction}

Ferroelectric (FE) materials are of great interest due to their broad range of next-generation which perhaps can be used in technological applications. FE materials have already been used in memory and storage devices [1-5] and are now being explored as components in sensors, actuators, and battery-related technologies [6-8]. FE functionalities and these FE functionalities involve composite creations that consist of small FE nanoparticles diffused in a dielectric matrix. We find that, on the other hand, FE nanoparticles can produce various shapes through synthetic techniques, such as spherical [9-11] and core-shell [12] geometries. However, the current studies of the effect of size and shape on FE properties have been largely focused on thin films [13-15], with few research studies dedicated to octahedral nanoparticles.
The system of competing energy interaction strengths, including electrostatics, domain wall, and electrostrictive coupling, depends on nanoparticle size and shape, material parameters, and the surrounding environment. Since some of these terms are of similar magnitude, the system becomes very sensitive to changes by control parameters.

Although the existence of vortex-like states in ferroelectric nanostructures, such as nanodots and nanowires, is well known, [16] until recently, these structures shapes were limited to simple rectilinear, cylindrical, or spherical geometries. However, we find that utilization of more elaborate nanoparticle shapes involving changing curvature and sharp geometrical features can result in distinctive new modes of functional behavior that may be particularly easy to control through external means.

We show that the general trend of transformations from monodomain, through vortex-like, to multidomain 
polarization texture holds for all the particle shapes as the size increases. However, the critical sizes at which the polarization pattern instabilities develop are strongly shape dependent, with octahedral particles undergoing their sequence of transitions at much larger sizes, compared to the spherical and cubic particles.

In this paper, we focus on the research of octahedral nanoparticles dispersed in a dielectric medium. The electrostatic interactions among individual octahedral nanoparticles can be ignored. We attempt to elucidate the size-dependent behaviors of the polar dipoles' equilibrium arrangement (polarization texture or pattern) in the isolated FE octahedral nanoparticles with varied materials parameters. We find that near-critical nanoparticle size between different polarization textures, a topological phase transformation, can be triggered by an applied electric.

Two archetypical FE materials, i.e., $\mathrm{PbTiO}_{3}$ (PT) and $\mathrm{BaTiO}_{3}(\mathrm{BT})$, are used to represent the properties of the octahedral nanoparticles. At room temperature, PT has a larger spontaneous polarization $\left(P_{\mathrm{s}}^{\mathrm{PT}}=0.75 \mathrm{C} \cdot \mathrm{m}^{-2}\right)$ than that of BT $\left(P_{\mathrm{s}}^{\mathrm{BT}}=0.26 \mathrm{C} \cdot \mathrm{m}^{-2}\right)$. However, between elastic strain and ferroelectric polarization, PT has weaker coupling than BT. Two materials with radically different dielectric are employed for the dielectric matrix: $\mathrm{SrTiO}_{3}$ (ST, high dielectric permittivity) and amorphous silica $\left(\mathrm{a}-\mathrm{SiO}_{2}\right.$, low dielectric permittivity). Our simulation results show that vortex-like polarization textures are superior to monodomain and multidomain patterns in a certain range of materials parameters and particle sizes. In some spatially confined FE nanostructures, similar polarization geometries have been observed experimentally [17-19] and predicted theoretically [20-25].

\section{Methods}

A three-dimensional finite-element approach is employed to compute the evolution of electrostatic potential $\boldsymbol{\Phi}$, elastic displacement fields $\boldsymbol{u}$, and coupled polarization density $\boldsymbol{P}$ in the system. Also, we used the FERRET code package, based on the Multiphysics Object-Oriented Simulation Environment (MOOSE) framework [26], to do all the numerical simulations. In this study, an octahedral FE inclusion $\left(\Omega_{\mathrm{FE}}\right)$ is selected, which is embedded in a nonpolarizable medium cube $\left(\Omega_{\mathrm{M}}\right)$ to represent an individual nanostructure for simulation. The inclusion and dielectric medium were meshed with an unstructured grid of tetrahedrons. The inclusion and dielectric medium were meshed with an unstructured grid of tetrahedrons. The core part and the outer part's interface are assumed to be coherent.

The system's total free energy in the domain $\Omega_{\mathrm{FE}}$ is given by the following expression by using the thermodynamic theory of Landau-Ginzburg-Devonshire (LGD):

$$
F=\int_{\Omega_{\mathrm{FE}}}\left[f_{\text {bulk }}+f_{\text {wall }}+f_{\text {elastic }}+f_{\text {elec }}+f_{\text {coupled }}\right] d \boldsymbol{V}
$$

Here, the factor $f_{\text {elastic }}$ represents the linear elastic energy density, the factor $f_{\text {elec }}$ represents the electrostatic energy density, and the factor $f_{\text {coupled }}$ represents the energy density due to strain and the local FE polarization density's electrostrictive coupling.

The polarization density field P's temporal evolution can be described by the following time-dependent LandauGinzburg-Devonshire (TDLGD) equation:

$$
-\gamma \frac{\partial \mathbf{P}}{\partial t}=\frac{\delta F}{\delta \mathbf{P}}
$$

where $\gamma$ is a time constant that has to do with polar domainwall mobility [27], $t$ is time, and $F$ is the total free energy of the system. We are disinterested in the system's temporal evolution and only interested in its final equilibrium state, so the time constant $\gamma$ is set to unity in this simulation. The TDLGD equation was used to reduce the total free energy of the system until it reaches a state of equilibrium. In this paper, we used a starting guess for all the particle sizes for the $P$ field by using a random paraelectric starting condition (RPEIC). Outside the FE inclusion is a nonpolarizable medium, i.e., $\mathbf{P} \equiv 0$, and the behavior of the medium is controlled by the linear elastic-dielectric medium equations. The electrostatic potential field $\Phi$ is calculated by the Poisson equation

$$
\nabla \cdot\left(\varepsilon_{\alpha} \nabla \Phi\right)=-\nabla \cdot \mathbf{P}
$$

And the condition for mechanical equilibrium is governed by

$$
\nabla \cdot \sigma=0
$$

It is always believed that the polarization density field's characteristic relaxation times are much slower than the elastic displacement fields and the electrostatic potential's characteristic relaxation times. Therefore, equations (3) and (4) have to be satisfied at each step in system time evolution governed by equation (2). Here, $\varepsilon_{\alpha}$ represents the constant of FE material's background dielectric $(\alpha=\mathrm{b})$ or the matrix dielectric constant $(\alpha=\mathrm{m})$, while $\sigma_{i j}=C_{i j k l} \partial u_{k} / \partial x_{l}$ represents the stress field of octahedral nanoparticles' local surface terminations.

Local surface terminations of the octahedral nanoparticles vary depending on the synthesis route. The terminations of the perovskite materials in this paper, composing in $\mathrm{TiO}$ or $\mathrm{Pb}(\mathrm{Ba}) \mathrm{O}$ layers would produce amounts of uncompensated surface charge differently, which will affect the polarization field distribution on the octahedral nanostructure surface. The charge compensation aggregate effects at surface terminations of the different octahedral nanoparticle can be captured by studying the dielectric constants of two different surrounding matrixes $\left(\mathrm{SrTiO}_{3}\right.$ and $\left.a-\mathrm{SiO}_{2}\right)$. The size of the cubic domain is considered large enough for the stresses $\sigma_{i j}$ and the elastic displacement field $\boldsymbol{u}$ created in an elastic mismatch at the interface between the matrix and the inclusion and vanishes at boundaries of the domain. Dirichlet boundary conditions $\boldsymbol{u}=0$ and $\boldsymbol{\Phi}=0$ are applied on the [ \pm 100$]$, $[0 \pm 10]$, and $[00 \pm 1]$ boundaries of the cubic domain. Additionally, a Dirichlet boundary condition, like $\Phi \neq 0$, can be employed on the [001] surface to introduce an external 


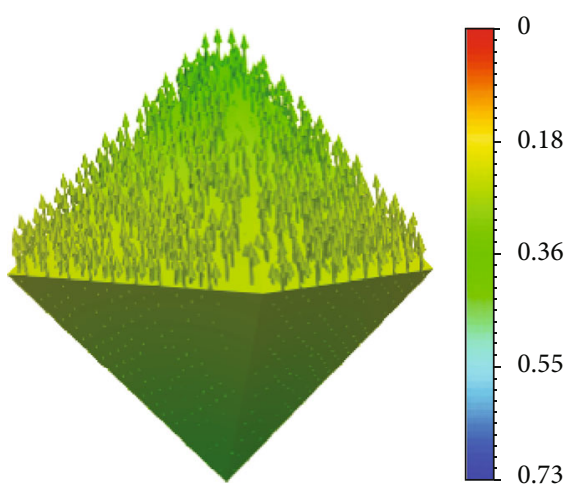

(a)

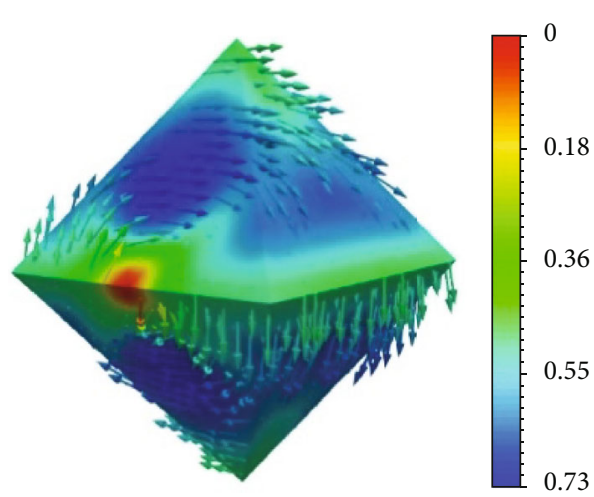

(b)

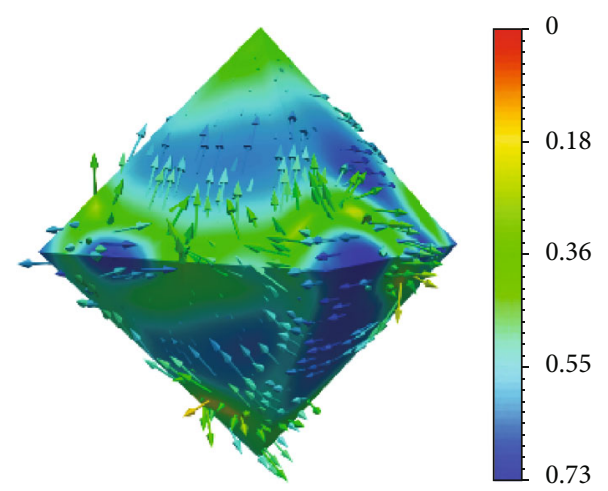

(c)

FIGURE 1: In the PT/ST system, as the sides length $l$ of the octahedral nanoparticles increase, various polarization patterns are observed: (a) monodomain, (b) vortex, and (c) multidomain. The local directions of the coupled polarization density $\boldsymbol{P}$ are indicated by arrows, and the color change indicated the field intensity change. The unit of polarization is $\mathrm{C} / \mathrm{m}^{2}$. The side lengths of the three octahedral particles are $1.1,6.5$, and $10.2 \mathrm{~nm}$.

electrostatic field. In our experimental calculation, all simulations are done at room temperature.

\section{Results and Discussion}

In this paper, the evolution of polarization textures in $\mathrm{FE}$ octahedral nanoparticles with changing particle size, applied electric field, and dielectric constant of the surrounding matrix was researched. Two distinct transitions of polarization textures, i.e., from a polar-monodomain or paraelectric to a vortex-like state, and then to a polydomain state, were identified in each system as the size of the octahedral FE inclusion increases. The polar-monodomain, vortex-like, and polydomain states are shown in Figure 1, and the side lengths of the three octahedral particles are 1.1, 6.5, and $10.2 \mathrm{~nm}$, respectively. Although the two transitions are common to the FE materials investigated here, their occurring particle sizes rest with the specific FE materials parameters.

3.1. Paraelectric and Ferroelectric States for Small l. The polarization of the FE octahedral nanoparticle will disappear, when its side length is below a certain critical value $l_{c}$. We found that the value of $l_{c}$ to a large extent depends on the option of materials parameters for the inclusion and the dielectric matrix. When the side length of the octahedral nanoparticle exceeds $l_{c}$, a nonzero polarization distribution $\boldsymbol{P}$ may exist in some form. The critical value $l_{\mathrm{c}}$ spans from 1 to $2.6 \mathrm{~nm}$ in all the systems considered here, which fall within an approximate range identified by other research groups [28-30]. It is also much smaller than the length $L$ of the FE correlation [31].

Simulations conducted for the PT/ST and BT/ST systems with side length $l \leq l_{c}$ can help us understand the details of the evolution towards an equilibrium configuration. The polarization produced by the RPEIC is inhomogeneous distributed, which makes the $f_{\text {wall }}$ energy term relatively large at the beginning of the simulation. In a small octahedral nanoparticle with side length $l<<L$, the polarization initially develops to a monodomain state, producing a surface charge density $q_{\mathrm{s}}=\boldsymbol{P} \cdot \boldsymbol{n}$; the factor $n$ in this formula represents the surface normal vector of the octahedral inclusion. However, the existence of the uncompensated surface charge $q_{\mathrm{s}}$ leads to a sharply augmented $f_{\text {elec }}$ energy term if the dielectric constant of the outside medium is not big enough to screen out the FE inclusion's surface charge.

To reduce $f_{\text {elec }}$ energy term, the magnitude of polarization is uniformly reduced until $\mathbf{P} \approx 0$, which results in the system's paraelectric state. For inclusions with PT side length $l<2.5 \mathrm{~nm}$ and dielectric constant $\varepsilon_{\mathrm{m}} \approx 300$ (i.e., ST matrix), a monodomain state is observed, with a uniformly 


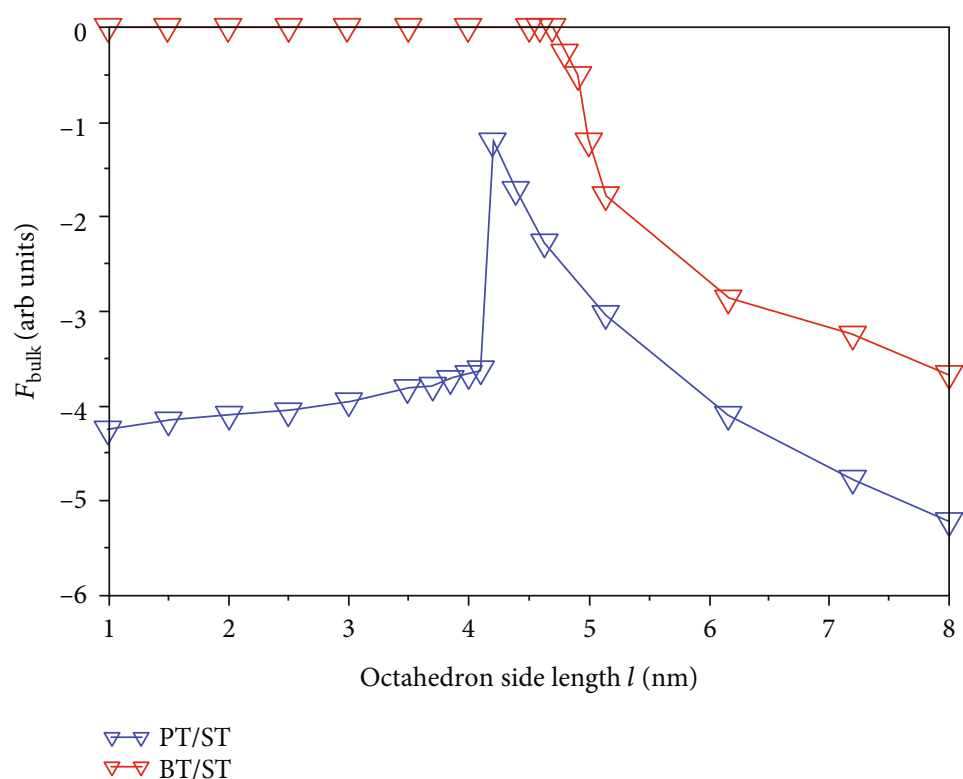

(a)

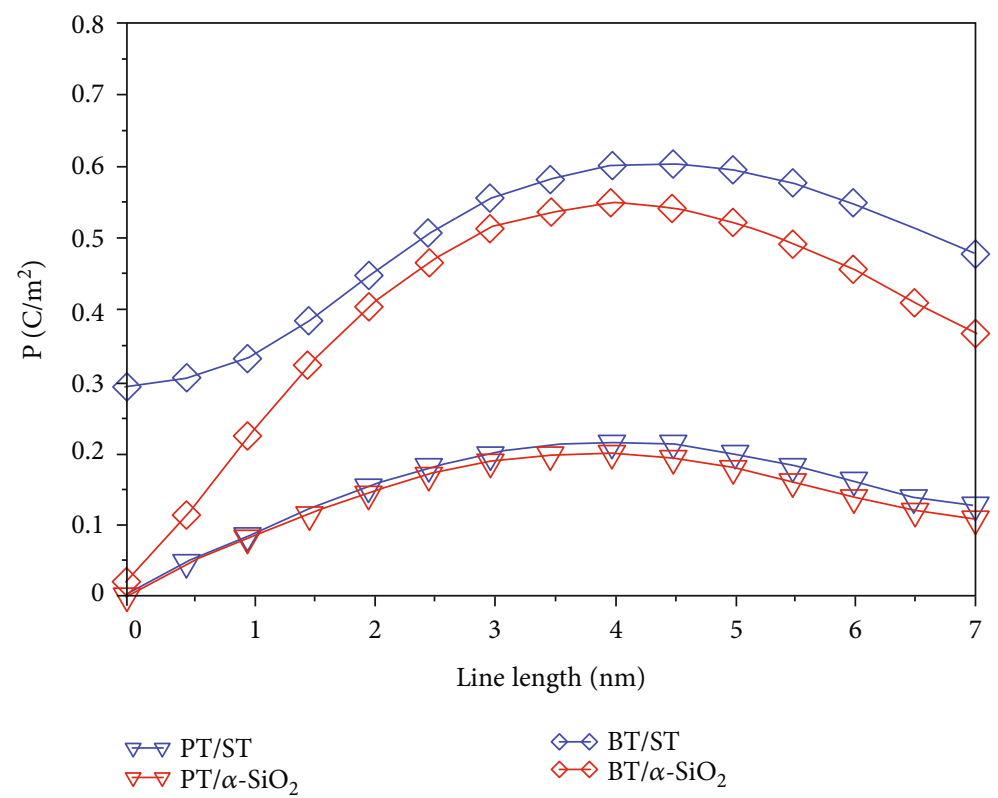

(b)

Figure 2: (a) The FE bulk energy ( $F_{\text {bulk }}$ ) of PT/ST and BT/ST systems plotted as a function of octahedral nanoparticle inclusion's side length $l$. (b) The profile of $|\boldsymbol{P}|$ along a line perpendicular to the vortex core in octahedral nanoparticles with vortex-like polarization textures.

distributed local polarization. For the lower values of dielectric constant $\varepsilon_{\mathrm{m}}$, we find a paraelectric state for the PT inclusion, while such state persists for ST inclusion with different values of $\varepsilon_{\mathrm{m}}$. This can be explained by stronger electrostrictive couplings and the shallower bulk energy minimum for the BT inclusion, which results in the energy growth that comes from the factor $f_{\text {bulk }}$ and the factor $f_{\text {coupled }}$ energy that was relatively quite small. The $f_{\text {elec }}$ energy term and minimization of $q_{\mathrm{s}}$ decided the movement toward equilibrium.

3.2. Transition into a Vortex-Like State. The vortex-like state's commencement in both BT and PT octahedral nano- particles are measurable and they can be measured by following normalized bulk free energy dependence,

$$
F_{\text {bulk }}=\frac{1}{\Omega_{\mathrm{FE}} P_{\mathrm{s}}^{3}} \int_{\Omega_{\mathrm{FE}}} f_{\text {bulk }} d \boldsymbol{V},
$$

on side length 1 which for the ST dielectric matrix is in Figure 1(b).

The topological phase transition from monodomain in ST/PT system to a vortex-like system occurs at a critical side length $l_{\mathrm{v}} \approx 2.5 \mathrm{~nm}$ accompanied by a sharp increase of $F_{\text {bulk }}$ 


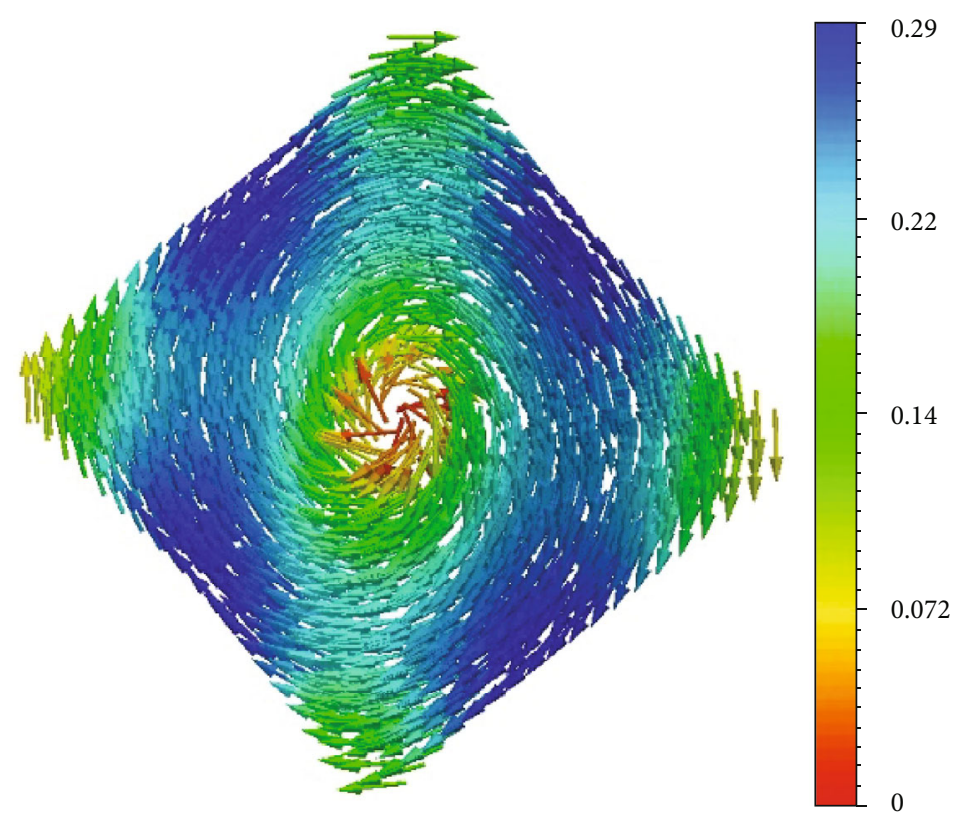

(a)
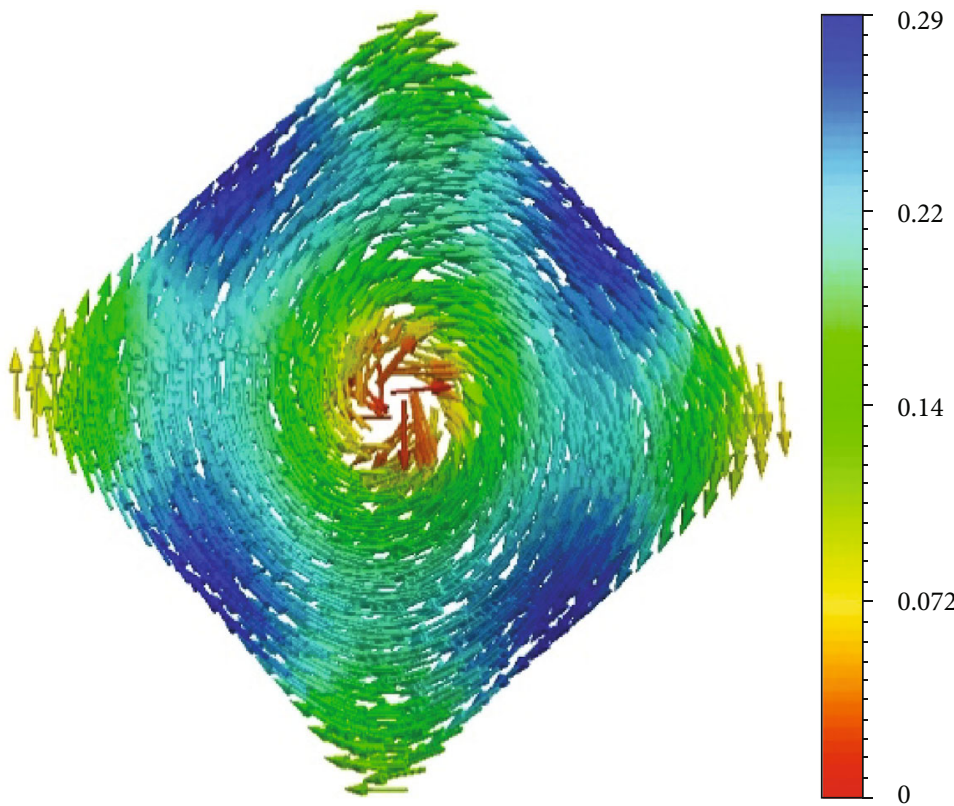

(b)

Figure 3: The vortex-like polarization textures in $\mathrm{BT} / \mathrm{ST}$ and $\mathrm{BT} / \mathrm{a}-\mathrm{SiO}_{2}$ octahedral nanoparticles with $l=5 \mathrm{~nm}$.

as shown in Figure 2(a). However, the monodomain state was not formed in the BT/ST system for $l \leq 3.6 \mathrm{~nm}$; instead, it stayed in the paraelectric state. Above that side length value, it transforms into a vortex-like state accompanied by an energy decrease. In the BT/ST system, the physics underpinning the energy change is easy to understand. To figure out the behavior in the ST/PT system, the polarization patterns before and after the transition, i.e., Figures 1(a) and 1(b), should be examined and compared. As shown in Figure 3, although the values of $|\mathbf{P}| \sim P_{\mathrm{s}}^{\mathrm{BT}}$ nearby the BT inclusion's surface show an intense depressed close to its center, it then forms a weakly polar core region.

The value of polarization along a line perpendicular to the vortex core axis is presented in Figure 2(b) for octahedral nanoparticles with $l=9.9 \mathrm{~nm}$ for both BT systems and PT systems which combine with two different considered dielectric matrices, i.e., $\mathrm{SrTiO}_{3}$ and $a-\mathrm{SiO}_{2}$. The polarization in the core region is suppressed by a factor of $3-5$ by a surface value comparison (in ST/PT) or even completely disappears in PT (in PT/a-SiO2 and always in BT). Such behavior is different from that of ferromagnetic vortices, which have a constant 


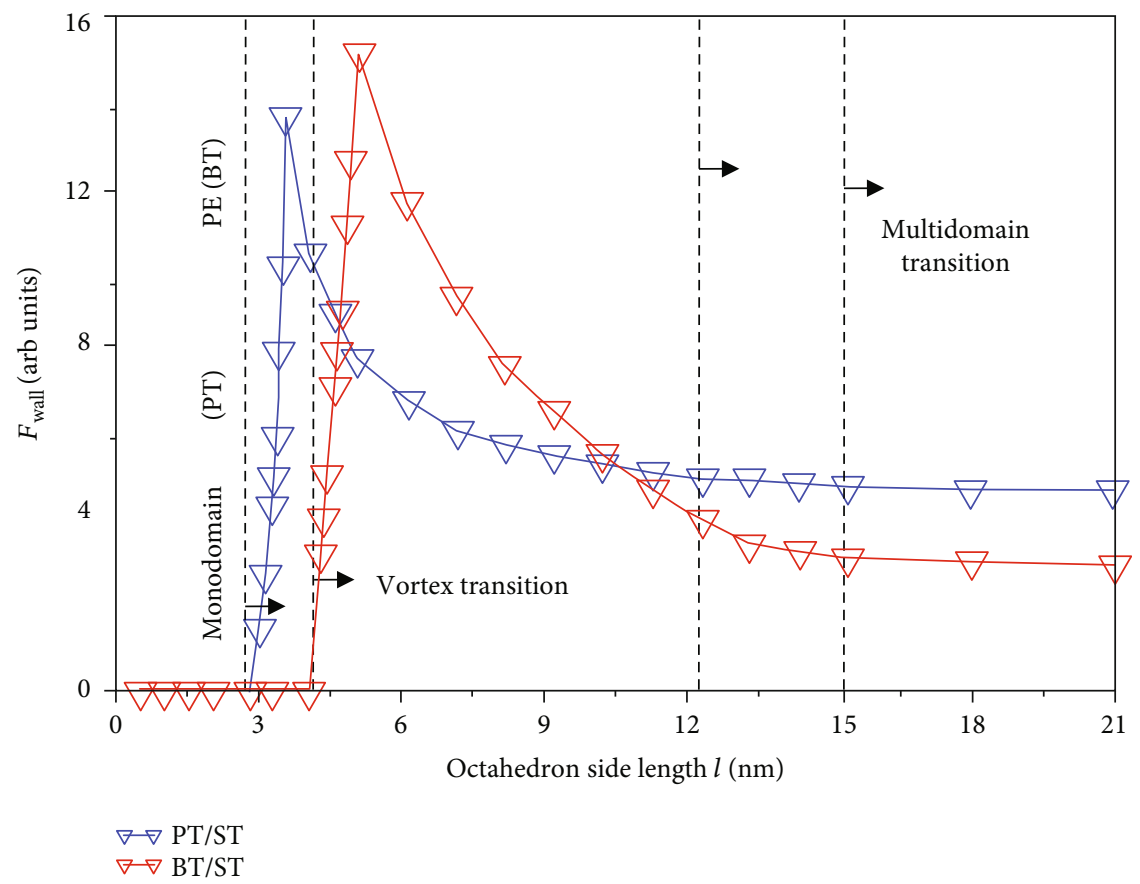

FIGURE 4: In PT/ST and BT/ST systems, $F_{\text {wall }}$ as a function of $\mathrm{d}$ and will be zero when $d<d_{\mathrm{v}}$. However, because of suboptimal arrangement of the local polarization vectors, normalized gradient energy $F_{\text {wall }}$ increased rapidly in the vortex-like state. It tends to be stable in the multidomain state, that is, it becomes a block, and its transition point (marked by a vertical line) is quite sensitively dependent on the choice of the $G_{i j k l}$ parameters for PT.

magnitude magnetization density at the core region. The influence of the surrounding matrix on the polarization values at the inclusion surface is also described in Figure 2(b). In the PT system, polarization at the surface is about one-fifth larger when the PT system is coupled with a high dielectric permittivity medium, i.e., ST. However, in the BT system, the polarization is not affected by the strength of the dielectric screening of the surrounding matrix.

3.3. Subsequent Transition into Multidomain State. When the side length of octahedral nanoparticle exceeds $l_{\mathrm{v}}$, the most important energy term influence on further polarization texture evolution is the normalized gradient energy.

$$
F_{\text {wall }}=\frac{1}{\Omega_{\mathrm{FE}} P_{\mathrm{s}}^{3}} \int_{\Omega_{\mathrm{FE}}} f_{\mathrm{wall}} d \boldsymbol{V} .
$$

In this paper, the parameterization set for the gradient energy tensor $G_{i j k l}$ in the Li et al. [32] was considered in the PT system. The well-known parameterization of Hlinka and Marton [33] was used for $G_{i j k l}$ in the BT system. In PT/ST and BT/ST systems, an energy term $F_{\text {wall }}$ dependence of 1 is shown in Figure 4. The value of the energy term in monodomain and paraelectric states is zero. It grows rapidly when the system transforms to a vortex-like state. This behavior can be explained by considering the vortex-like state as a state consisting of many domain walls, which separate small polar regions that have suboptimal polarization arrangements. As the octahedral nanoparticle side length increases beyond $l_{v}$, the energy term $F_{\text {wall }}$ gradually recedes to a steady nonzero value. The leveling off of $F_{\text {wall }}$ indicates a multidomain state formation, which is composed of relatively large areas of correlated polarization divided by domain walls. This phase transition happens at $l=l_{m}=12.3$ $\mathrm{nm}$ and $15.4 \mathrm{~nm}$ for the PT/ST and BT/ST system, respectively.

The multidomain polarization textures are depicted in Figure 1(c). To reduce the electrostatic energy produced by $q_{s}$, domains orient their polarization direction tangentially to the surface of octahedral nanoparticles. The weakly polar vortex core region in the octahedral nanoparticle becomes unstable at side length $l>l_{m}$, partitioning into multiple domains. The polarization texture vorticity diminishes sharply after the phase transition from vortex-like to multidomain state; however, it does not completely disappear but continues as localized vortices which still remain nearby some domain walls, which results in finite values of $F_{\text {wall }}$ at large side length $l$.

Due to the normal vector of the octahedral nanoparticle, surfaces have different orientations; the domains cannot be directly split into low energy $90^{\circ}$ or $180^{\circ}$ variants collections when the position is in the near-surface region.

\section{Conclusions}

In this article, we simulated the octahedral ferroelectric nanomaterials in different environments as different dielectric constants in different dielectric matrices and obtained the material's electrostrictive energy, electrostatic charge, and domain wall evolution results. In general, we found that the dielectric response of ferroelectric octahedral 
nanomaterials can be changed by changing the material parameters by using different materials or by adjusting the external electric field. At the same time, the excitation results show that PT octahedral nanoparticles can generate a nonzero polarization field in a dielectric matrix with a high dielectric constant and so can generate compensation charges on the surface of the octahedron. In contrast, when the FE particle size is lower than the critical value $l_{\nu}$, due to the existence of a large amount of uncompensated charges on the surface, the polarization of the ferroelectric material will be affected by the PT octahedral nanoparticle in a dielectric matrix with a low dielectric constant. When the size is greater than the critical value $l_{v}$, the electrostrictive energy of the material is minimized due to the existence of the surface charge; therefore, the ferroelectric particles will produce vortex domain walls. In larger sizes, since the polarization energy and electrostrictive energy in the total free energy of the system are balanced, the ferroelectric particles exhibit a multidomain-like domain wall pattern.

Understanding and controlling the collective behavior of both ordered and disordered assemblies of such particles, embedded into a variety of dielectric environments, [34, 35] is paramount for the engineering of advanced functional properties, not present in the bulk or other nanoscopic phases (e.g., thin films) of the parent ferroelectric compounds. For example, $\mathrm{BaTiO}_{3}$ (BTO) nanoparticles dispersed in parylene exhibit superparaelectric behavior [34] that could be exploited for energy storage applications, while their ordered superlattices display distinct hysteretic switching patterns depending on the size and packing order [36].

\section{Data Availability}

All data used to support the findings of this study are included within the article.

\section{Conflicts of Interest}

The authors declare that they have no conflicts of interest.

\section{Acknowledgments}

The authors acknowledge the financial support from the Science and technology research project of education department of Jiangxi Province in 2019 (Grant No. GJJ190497) and the research project for high-level talents in Jiangxi University of Science and Technology (Grant No. jxncbs19009).

\section{References}

[1] V. Presser, C. R. Dennison, J. Campos, K. W. Knehr, E. C. Kumbur, and Y. Gogotsi, "The electrochemical flow capacitor: a new concept for rapid energy storage and recovery," Advanced Energy Materials, vol. 2, no. 7, pp. 895-902, 2012.

[2] A. Verma, A. K. Yadav, N. Khatun et al., "Corrigendum to "Structural, dielectric and ferroelectric studies of thermally stable and efficient energy storage ceramic materials: $\left(\mathrm{Na}_{0.5-}\right.$ $\left.{ }_{x} \mathrm{~K}_{\mathrm{x}} \mathrm{Bi}_{0.5-\mathrm{x}} \mathrm{La}_{\mathrm{x}}\right) \mathrm{TiO}_{3}$ " [Ceram. Int. 44 (2018) 20178-20186]," Ceramics International, vol. 45, no. 10, pp. 13605-13605, 2019.
[3] P. Zhao, H. Wang, L. Wu et al., "High-performance relaxor ferroelectric materials for energy storage applications," Advance Energy Materials, vol. 9, no. 17, article 1803048, 2019.

[4] G. Prando, "Walls and memory," Nature Nanotechnology, vol. 12, no. 8, pp. 724-724, 2017.

[5] M. Vopson, G. Caruntu, and X. Tan, "Polarization reversal and memory effect in anti-ferroelectric materials," Scripta Materialia, vol. 128, pp. 61-64, 2017.

[6] B. Stadlober, M. Zirkl, and M. Irimia-Vladu, "Route towards sustainable smart sensors: ferroelectric polyvinylidene fluoride-based materials and their integration in flexible electronics," Chemical Society Reviews, vol. 48, no. 6, pp. 17871825, 2019.

[7] R. Gellmann and A. Ricoeur, "Continuum damage model for ferroelectric materials and its application to multilayer actuators," Smart Materials and Structures, vol. 25, no. 5, article 055045, 2016.

[8] J. D. B. Wu, J. D. Burton, E. Y. Tsymbal, X. C. Zeng, and P. Jena, "Hydroxyl-decorated graphene systems as candidates for organic metal-free ferroelectrics, multiferroics, and highperformance proton battery cathode materials," Physical Review B, vol. 87, no. 8, article 081406, 2013.

[9] F. Di Rino, M. Sepliarsky, and M. G. Stachiotti, “Topology of the polarization field in $\mathrm{PbTiO} 3$ nanoparticles of different shapes by atomic-level simulations," Journal of Applied Physics, vol. 127, no. 14, p. 144101, 2020.

[10] I. H. Lone, A. Kalam, J. Ahmed, N. Alhokbany, S. M. Alshehri, and T. Ahmad, "Quenching assisted reverse micellar synthesis and electrical properties of high surface area BiFeO3Nanoparticles," Journal of Nanoscience and Nanotechnology, vol. 20, no. 6, pp. 3823-3831, 2020.

[11] L. Yang, Q. Zhao, K. Chen et al., "Simultaneously realizing ultra-high energy density and discharge efficiency in PVDF composites loaded with highly aligned hollow $\mathrm{MnO}_{2}$ microspheres," Composites Part A-Applied Science and Manufacture, vol. 132, article 105820, 2020.

[12] S. L. Kang, F. Wang, X. L. Zhong, J. B. Wang, and B. Li, "Barium titanate/poly (vinylidene fluoride) nanocomposites with core-shell structure with high dielectric constant and temperature stability prepared via a cold sintering process," Journal of Materials Science: Materials in Electronics, vol. 31, no. 12, pp. 9284-9292, 2020.

[13] J. Zhao, G. Niu, W. Ren et al., "Structural and electrical properties of sodium bismuth titanate based 0-3 composite leadfree ferroelectric thick films," Journal of Alloys and Compounds, vol. 829, p. 154506, 2020.

[14] M. D. Glinchuk, A. N. Morozovska, A. Lukowiak et al., "Possible electrochemical origin of ferroelectricity in $\mathrm{HfO}_{2}$ thin films," Journal of Alloys and Compounds, vol. 830, no. 25, article $153628,2020$.

[15] P. P. Biswas, S. Pal, V. Subramanian, and P. Murugavel, "Polarization driven self-biased and enhanced UV-visible photodetector characteristics of ferroelectric thin film," Journal of Physics D-Applied Physics, vol. 53, no. 27, p. 275302, 2020.

[16] J. M. Gregg, "Exotic domain states in ferroelectrics: searching for vortices and skyrmions," Ferroelectrics, vol. 433, no. 1, pp. 74-87, 2012.

[17] A. Gruverman, D. Wu, H.-J. Fan et al., "Vortex ferroelectric domains," Journal of Physics: Condensed Matter, vol. 20, no. 34, article 342201, 2008. 
[18] Z. Hong, S. Das, C. Nelson et al., "Vortex Domain Walls in Ferroelectrics," Nano Letters, vol. 21, no. 8, pp. 3533-3539, 2021.

[19] S. C. Chae, N. Lee, Y. Horibe et al., "Direct Observation of the Proliferation of Ferroelectric Loop Domains and VortexAntivortex Pairs," Physical Review Letters, vol. 108, no. 16, article 167603, 2012.

[20] I. I. Naumov, L. Bellaiche, and H. Fu, "Unusual phase transitions in ferroelectric nanodisks and nanorods," Nature, vol. 432, no. 7018, pp. 737-740, 2004.

[21] I. Ponomareva, I. I. Naumov, I. Kornev, H. Fu, and L. Bellaiche, "Atomistic treatment of depolarizing energy and field in ferroelectric nanostructures," Physical Review B, vol. 72, no. 14, article 140102, 2005.

[22] I. Naumov and A. M. Bratkovsky, "Unusual polarization patterns in flat epitaxial ferroelectric nanoparticles," Physical Review Letters, vol. 101, no. 10, p. 107601, 2008.

[23] A. P. Levanyuk and R. Blinc, "Ferroelectric phase transitions in small particles and local regions," Physical Review Letters, vol. 111, no. 9, article 097601, 2013.

[24] P. W. Martelli, S. Mefire, and I. A. Luk'yanchuk, "Multidomain switching in the ferroelectric nanodots," Europhysics Letters, vol. 111, no. 5, article 50001, 2015.

[25] Y. Nahas, S. Prokhorenko, L. Louis, Z. Gui, I. Kornev, and L. Bellaiche, "Discovery of stable skyrmionic state in ferroelectric nanocomposites," Nature Communications, vol. 6, no. 1, p. $8542,2015$.

[26] D. Gaston, C. Newman, G. Hansen, and D. Lebrun-Grandié, "MOOSE: a parallel computational framework for coupled systems of nonlinear equations," Nuclear Engineering and Design, vol. 239, no. 10, pp. 1768-1778, 2009.

[27] Q. Meng, M. G. Han, J. Tao, G. Xu, D. Welch, and Y. Zhu, "Velocity of domain-wall motion during polarization reversal in ferroelectric thin films: beyond Merz's law," Physical Review $B$, vol. 91, no. 5, article 054104, 2015.

[28] D. D. Fong, G. B. Stephenson, S. K. Streiffer et al., "Ferroelectricity in ultrathin perovskite films," Science, vol. 304, no. 5677, pp. 1650-1653, 2004.

[29] E. Erdem, H. Semmelhack, R. Bottcher et al., "Study of the tetragonal-to-cubic phase transition in $\mathrm{PbTiO} 3$ nanopowders," Journal of Physics: Condensed Matter, vol. 18, no. 15, p. 3861, 2006.

[30] A. Grünebohm, M. E. Gruner, and P. Entel, "Domain structure in the tetragonal phase of $\mathrm{BaTiO}_{3}$-from bulk to nanoparticles," Ferroelectrics, vol. 426, no. 1, pp. 21-30, 2012.

[31] M. D. Glinchuk, E. A. Eliseev, and A. N. Morozovska, "Superparaelectric phase in the ensemble of noninteracting ferroelectric nanoparticles," Physical Review B, vol. 78, no. 13, p. 134107, 2008.

[32] Y. L. Li, S. Y. Hu, Z. K. Liu, and L. Q. Chen, "Effect of electrical boundary conditions on ferroelectric domain structures in thin films," Applied Physics Letters, vol. 81, no. 3, pp. 427-429, 2002.

[33] J. Hlinka and P. Márton, "Phenomenological model of a $90^{\circ}$ domain wall in $\mathrm{BaTiO}_{3}$-type ferroelectrics," Physical Review $B$, vol. 74, no. 10, p. 104104, 2006.

[34] L. Huang, Z. Jia, I. Kymissis, and S. O'Brien, "HighKCapacitors and OFET gate dielectrics from self-assembled BaTiOzand (Ba,Sr)TiO3Nanocrystals in the superparaelectric limit," Advanced Functional Materials, vol. 20, no. 4, pp. 554-560, 2010.
[35] S. A. Paniagua, Y. Kim, K. Henry, R. Kumar, J. W. Perry, and S. R. Marder, "Surface-initiated polymerization from barium titanate nanoparticles for hybrid dielectric capacitors," ACS Applied Materials \& Interfaces, vol. 6, no. 5, pp. 3477-3482, 2014.

[36] J. Feng, Q. Song, B. Zhang, Y. Wu, T. Wang, and L. Jiang, "Large-scale, long-range-ordered patterning of nanocrystals via capillary-bridge manipulation," Advanced Materials, vol. 29, article 1703143, no. 46, 2017. 